\title{
Clinical utility gene card for: Hereditary thrombocythemia
}

\author{
Kais Hussein ${ }^{\star, 1,13}$, Melanie Percy ${ }^{2,13}$, Mary Frances McMullin ${ }^{3,13}$, Jiří Schwarz ${ }^{4,13}$, Susanne Schnittger ${ }^{5,13}$, \\ Naomi Porret ${ }^{6,13}$, Luz Maria Martinez-Aviles ${ }^{7,13}$, Beatriz Bellosillo Paricio ${ }^{7,13}$, Stéphane Giraudier ${ }^{8,13}$, \\ Radek Skoda $^{9,13}$, Eric Lippert ${ }^{10,13}$, Sylvie Hermouet ${ }^{11,13}$ and Holger Cario ${ }^{12,13}$
}

European Journal of Human Genetics (2014) 22, doi:10.1038/ejhg.2013.117; published online 5 June 2013

\section{DISEASE CHARACTERISTICS}

1.1 Name of the disease (synonyms)

Hereditary thrombocythemia (congenital or familial thrombocytosis or essential thrombocythemia).

\subsection{OMIM\# of the disease}

187950 (Thrombocythemia 1/THCYT1, autosomal dominant); 601977 (thrombocythemia 2/THCYT2, autosomal dominant and autosomal recessive); 614521 (thrombocythemia 3/THCYT3, autosomal dominant with incomplete penetrance); 300331 (thrombocythemia, X-linked, THCYTX, autosomal recessive).

Note that the OMIM designation of the thrombocythemia types have been changed in 2012 following 'erythrocytosis, familial 1-4 (ECYT1-4)': 187950 was formerly 'essential thrombocythemia' (now THCYT1) and 601977 was formerly 'benign familial microcytic thrombocytosis' (now THCYT2).

1.3 Name of the analyzed genes or DNA/chromosome segments Thrombopoietin (THPO)/3q27; myeloproliferative leukemia virus oncogene (MPL, synonym thrombopoietin receptor)/1p34; Janus kinase $2(J A K 2) / 9 q 24$.

1.4 OMIM\# of the gene(s)

600044 (THPO); 159530 (MPL); 147796 (JAK2).

\subsection{Mutational spectrum}

THPO (THCYT1): IVS3, G-C, +1 (G to $\mathrm{C}$ transversion at position +1 of intron 3). ${ }^{1-3}$ 1-BP DEL, 3252G (1-bp G deletion in the $5^{\prime}$ untranslated region at nucleotide position 3252 and localization of deleted G 47 bases upstream of the authentic initiation codon). ${ }^{4,5}$ G516T of the $5^{\prime}$-untranslated region. ${ }^{6,7}$ Mutations of THPO signallingassociated factors other than MPL and JAK2 (eg, SH2B adaptor protein 3/SH2B3) have not yet been reported in hereditary cases.
MPL (THCYT2): G1238T/K39N (germline alteration, 'MPL Baltimore'). ${ }^{8}$ G317T/P106L (germline alteration). ${ }^{9}$ G1073A/S505N (germline alteration). ${ }^{10-12}$ Codon W515 mutations have not yet been reported in hereditary cases but in sporadic essential thrombocythemia (ET). ${ }^{13}$

JAK2 (THCYT3): G1894T/V617F (somatic mutation in THCYT3 and sporadic ET). ${ }^{14,15}$ G1894A/V617I (germline mutation in THCYT3). ${ }^{16}$ The molecular pathology of THCYTX $^{17}$ and benign familial microcytic thrombocytosis is not known. ${ }^{18}$

\subsection{Analytical methods}

Sequencing of peripheral blood or bone marrow leukocytes (DNA and/or RNA/cDNA).

Restriction analyses:

THPO IVS3, G-C, +1 : the mutation abolishes a BsrI restriction endonuclease recognition site. ${ }^{2}$ MPL G1238T mutation: the mutation creates a Tsp509I restriction endonuclease cleavage site. ${ }^{8}$ MPL G317T mutation: the mutation abolishes an Acil restriction endonuclease recognition site. ${ }^{9}$ JAK2 G1894T mutation: the mutation abolishes a BsaXI restriction endonuclease recognition site. ${ }^{19}$

\subsection{Analytical validation}

Bidirectional sequencing, double measurements.

Control samples: mutated patient sample (if available), unmutated patient sample and/or cell lines (eg, JAK2 G1894T-positive leukemia cell lines are HEL, MB-02, MUTZ-8, UKE-1 and SET2). As yet, THPO or MPL-mutated cell lines are not known.

1.8 Estimated frequency of the disease

(incidence at birth ('birth prevalence') or population prevalence) Population prevalence: $<1: 100.000 .^{20,21}$

1.9 If applicable, prevalence in the ethnic group of investigated person

The exact overall prevalence is not known, but likely $<1: 100.000 .^{20,21}$

${ }^{1}$ Institute of Pathology, Hannover Medical School, Hannover, Germany; ${ }^{2}$ Department of Hematology, Belfast City Hospital, Belfast, UK; ${ }^{3}$ Department of Hematology, Queen's University, Belfast, UK; ${ }^{4}$ Institute of Hematology and Blood Transfusion, Prague, Czech Republic; ${ }^{5}$ MLL Munich Leukemia Laboratory, Munich, Germany; ${ }^{6}$ Department of Hematology, Inselspital, Bern, Switzerland; ${ }^{7}$ Department of Pathology, Hospital del Mar Barcelona, Barcelona, Spain; ${ }^{8}$ Department of Hematology, Hopital H Mondor AP-HP Paris, Paris, France; 9 University Hospital Basel, Basel, Switzerland; 10 University Hospital, Bordeaux, France; ${ }^{11}$ Inserm UMR892/CNRS UMR6299, Centre de Recherche en Cancérologie Nantes-Angers, Institut de Recherche en Santé, Université de Nantes, Nantes, France; ${ }^{12}$ Department of Pediatrics and Adolescent Medicine, University Medical Center Ulm, Ulm, Germany

${ }^{13}$ Members of COST Action BM0902 (MPN\&MPNr-EuroNet).

*Correspondence: Dr K Hussein, Institute of Pathology, Hannover Medical School, Carl-Neuberg-Street 1, 30625 Hannover, Germany. Tel: + 4905115324501 ;

Fax: + 490511532 5799; E-mail: Hussein.Kais@mh-hannover.de 
In principle, individuals from any ethnic group can develop THCYT/hereditary ET.

Hereditary thrombocythemia cases with associated THPO alterations (THCYT1) have been described in Japan, ${ }^{4,6,7}$ Italy, ${ }^{5}$ the Netherlands ${ }^{1,2}$ and Poland. ${ }^{3}$

In an affected Italian family with THPO 1-BP DEL, 3252G distal unilateral limb defects were present; presumably secondary defects (grandfather without limb defects; father with absence of right forearm and hand and absence of right foot, one of three male children with absence of the right foot, the calcaneus and astragalus; a second child with absence of the last phalange of 2 nd digit and last 2 phalanges of 3 rd to 5 th digits; the third child had no limb defects). ${ }^{5}$

Hereditary thrombocythemia cases with associated $M P L$ alterations (THCYT2) have been described in Japan, ${ }^{10}$ Italy ${ }^{11,12}$ North America (African Americans) ${ }^{8}$ and Arabia. ${ }^{9}$

Regional/ethnic association is known for MPL K39N ( $\sim 7 \%$ of African Americans are heterozygous for MPL K39N, $n=12 / 161)$ and MPL P106L $(\sim 5 \%$ in Arab population, $n=11 / 213 ; \sim 1 \%$ are homozygous and $\sim 4 \%$ are heterozygous for MPL P106L). The frequency of MPL K39N in other American ethnics is $0 \%$ (Caucasian, $n=0 / 250$; Hispanic, $n=0 / 40$; Asian, $n=0 / 39$ ). The frequency of MPL P106L in German individuals is $0 \%(n=0 / 193){ }^{8,9}$ As yet, MPL S505N has not been systematically evaluated regarding regional/ethnic association.

Hereditary thrombocythemia cases with associated JAK2 alterations (THCYT3) have been described in a cohort of 66 European and 6 African families with somatic JAK2 V617F mutation $^{15}$ and germline JAK2 V617I was detected in a family from Great Britain. ${ }^{16}$

Data from Sweden revealed that relatives of ET patients had elevated risk for any myeloproliferative neoplasm (MPN; $\mathrm{RR}=6.8 ; 95 \% \mathrm{CI}, 3.4-13.5)$, $\mathrm{ET}(\mathrm{RR}=8.8 ; 95 \% \mathrm{CI}, 2.6-24.5)$, Polycythemia vera $(\mathrm{RR}=5.4 ; 95 \% \mathrm{CI}, 2.2-13.1)$ and unclassifiable MPN $(\mathrm{RR}=16.1 ; 95 \% \quad \mathrm{CI}, 1.9-146.1)$ but no significantly increased risk for primary myelofibrosis (PMF; $\mathrm{RR}=4.0 ; 95 \%$ CI, $0.2-61.7) .^{22}$

THCYTX has been described in an Arab family from Saudi Arabia. ${ }^{17}$

Benign familial microcytic thrombocytosis has been described in a Jewish family (mother with disease phenotype emigrated from India). ${ }^{18}$

\subsection{Diagnostic setting}

\begin{tabular}{lll}
\hline & Yes & No \\
A. (Differential) diagnostics & $\Downarrow$ & $\square$ \\
B. Predictive testing & $\Downarrow$ & $\square$ \\
C. Risk assessment in relatives & $\bigotimes$ & $\square$ \\
D. Prenatal & $\square$ & $\square$
\end{tabular}

\section{Comment:}

Prenatal testing is not indicated in THCYT1-3 (even if limb defects are sonographically present) because the result of a genetic test has no consequence during pregnancy (detection of an associated mutation is no indication for induced abortion); the child of an affected mother can be tested after birth.

\section{TEST CHARACTERISTICS}

\begin{tabular}{|c|c|c|c|c|}
\hline & \multicolumn{2}{|c|}{ Genotype or disease } & \multirow{2}{*}{$\begin{array}{l}\text { A: True positives } \\
\text { B: False positives }\end{array}$} & \multirow{2}{*}{$\begin{array}{l}\text { C: False negative } \\
\text { D: True negative }\end{array}$} \\
\hline & Present & Absent & & \\
\hline \multicolumn{5}{|l|}{ Test } \\
\hline \multirow[t]{2}{*}{ Positive } & $A$ & B & Sensitivity: & $A /(A+C)$ \\
\hline & & & Specificity: & $D /(D+B)$ \\
\hline \multirow[t]{2}{*}{ Negative } & C & $\mathrm{D}$ & Positive predict. value: & $A /(A+B)$ \\
\hline & & & Negative predict. value: & $D /(C+D)$ \\
\hline
\end{tabular}

\subsection{Analytical sensitivity}

(proportion of positive tests if the genotype is present)

More than 99\% (False-negative rate $<1 \%$ ).

\subsection{Analytical specificity}

(proportion of negative tests if the genotype is not present)

More than 99\% (false-positive rate $<1 \%$ ).

\subsection{Clinical sensitivity}

(proportion of positive tests if the disease is present)

The clinical sensitivity can be dependent on variable factors such as age or family history. In such cases, a general statement should be given, even if a quantification can only be made case by case.

The clinical sensitivity is undetermined but likely $<50 \%$ of cases with thrombocythemia and positive family anamnesis are positive for one of the associated mutations.

\subsection{Clinical specificity}

(proportion of negative tests if the disease is not present)

The clinical specificity can be dependent on variable factors such as age or family history. In such cases, a general statement should be given, even if a quantification can only be made case by case.

The clinical specificity is undetermined but likely $>99 \%$ of cases with no thrombocythemia are negative for associated mutations.

\subsection{Positive clinical predictive value}

(life-time risk to develop the disease if the test is positive)

Autosomal recessive MPL P106L-positive THCYT2: $<25 \%$ in heterozygously mutated cases (only a small subfraction of heterozygous carriers develop thrombocythemia) and 100\% in homozygously mutated cases. ${ }^{9}$

Autosomal-dominant THCYT1, non-MPL P106L THCYT2 and THCYT3: $100 \%$ in heterozygously and homozygously mutated cases. $^{20,21}$

\subsection{Negative clinical predictive value}

(probability of not developing the disease if the test is negative) Assume an increased risk based on family history for a non-affected person. Allelic and locus heterogeneity may need to be considered.

The negative clinical predictive value is undetermined, because $>50 \%$ of cases with thrombocythemia and positive family anamnesis are negative for one of the associated mutations.

Index case in that family had been tested:

If the index patient is carrying a mutation, the risk of mutationnegative first-degree relatives to develop the disease is $0 \%$.

Index case in that family had not been tested:

If the index patient has not been tested for thrombocythemiaassociated mutations, the risk of non-affected first-degree relatives to 
develop the disease depends on recessive or dominant transmission of the disease:

Autosomal recessive MPL P106L-positive THCYT2, risk to develop the disease: $<25 \%$ if only one parent is a heterozygous carrier and the other parent is not (only a small subfraction of heterozygous carriers develop thrombocythemia), $25 \%$ if both parents are heterozygous carriers, $50 \%$ if one parent is a heterozygous carrier and one parent is a homozygous patient, $100 \%$ if both parents are homozygous patients. ${ }^{2}$

Autosomal-dominant THCYT1, non-MPL P106L THCYT2 and THCYT3, risk to develop the disease: $50 \%$ if one parent is a heterozygous patient and the other parent is not, $75 \%$ if both parents are heterozygous patients, $100 \%$ if one parent is a heterozygous patient and one parent is a homozygous patient, $100 \%$ if both parents are homozygous patients. ${ }^{20,21}$

\section{CLINICAL UTILITY}

3.1 Differential diagnostics: The tested person is clinically affected (To be answered if in 1.10 'A' was marked)

\subsubsection{Can a diagnosis be made other than through a genetic test?}

\begin{tabular}{lll}
\hline No & $\square$ (continue with 3.1.4) & \\
Yes & $\bigotimes$ & $\bigotimes$ \\
& Clinically & \\
Imaging & $\square$ \\
Endoscopy & $\bigotimes$ \\
Biochemistry & $\square$ \\
Electrophysiology & $\bigotimes$ \\
Other: & Anamnesis. \\
& Peripheral blood analysis: evaluation of \\
& hematological parameters.
\end{tabular}

Comment:

A thrombocythemia is present when the platelet count is chronically elevated $>450 \times 10^{9} / \mathrm{l}$. By definition, it is congenital if it is present from birth. In such persons the thrombocythemia is usually detected at a young age. There can be a family history in keeping with the inherited nature of the defect. However, sporadic cases without positive family history can occur.

Hereditary thrombocythemia can be primary or secondary: $:^{20,21}$

A primary thrombocythemia in terms of THCYT2 and THCYT3 occurs when there is an intrinsic defect in the megakaryocytic cells in the bone marrow (MPL and/or JAK2 mutations). In these cases, THPO levels will be normal (which would be inappropriate for a raised platelet count) or below normal.

A secondary thrombocythemia is present when THPO production in the liver (main source of THPO production), kidney and bone marrow stromal cells is increased. In case of THCYT1, mutation in the $5^{\prime}$-untranslated region of THPO leads to disturbed regulation of translation with increased THPO production.

Hereditary thrombocythemia can be non-neoplastic (THCYT1 and THCYT2) or neoplastic (THCYT3):

THCYT1 and THCYT2 have no elevated risk to develop acute leukemia; these cases are positive for THPO mutations or MPL mutations K39N or S505N and negative for MPL G1544T/W515L and MPL T1543A-G1544A/W515K (MPL W515L/K mutations can be found in sporadic ET), JAK2 V617F and TET2 mutations. ${ }^{11,20,22,23}$ Sporadic ET but not hereditary thrombocythemia with MPL codon W515 mutations have not been described so far. ${ }^{13}$
Adult and elderly patients, but not children with MPL S505Npositive THCYT2, have frequently developed myelofibrosis. ${ }^{11}$ These cases are indistinguishable from other BCR-ABL-negative PMF. Neoplastic THCYT3/hereditary ET have a low but nevertheless elevated risk to develop myelofibrosis and/or acute leukemia. ${ }^{20,21}$ THCYT3 can be positive for somatic JAK2 V617F ${ }^{15}$ or germline JAK2 V617I (reported so far in one family). ${ }^{16}$

Note that: MPL W515L/K mutations have been described in sporadic ET but as yet not in THCYT2. Without a positive family anamnesis THCYT3/hereditary ET and sporadic ET are indistinguishable. No detectable JAK2 mutation does not exclude hereditary ET because $\sim 50 \%$ of cases can be JAK2 V617F-negative and JAK2 V617I has been reported so far in only one family. ${ }^{15,16}$ Diagnosis of THCYT3/ hereditary ET in an index patient does not necessarily mean that other related patients must have an THCYT3/ET phenotype; it is known that different entities of MPN might present in individual patients of a family (hereditary MPN with individual ET manifestation). ${ }^{13,15,20,21}$ Diagnosis of THCYTX requires exclusion of all other THCYT subtypes. THPO IVS3, G-C, +1 mutation has been excluded in THCYTX but not other THPO mutations or mutations of MPL and JAK2. The serum THPO level has not been determined. ${ }^{17}$ Cytogenetic aberrations are thought to be rare in sporadic ET $(<10 \%$ of cases) and, if present, unspecific. Recurrent cytogenetic aberrations have not been reported in THCYT subtypes.

Consider screening those with (Figure 1): True thrombocythemia ( $>6$ months and $>450 \times 10^{9} / 1$ platelets), no identifiable secondary cause, young patients and/or positive family history.

Diagnosis of hereditary thrombocythemia: ${ }^{19,20}$

Anamnesis: positive family anamnesis and thromboembolic complications.

Physical examination and exclusion of secondary (reactive) cause of thrombocythemia: exclusion of acute/chronic infection $(\sim 50 \%$ of thrombocythemia cases are associated with infection), iron deficiency, acute blood loss, hemolysis, asplenia, paraneoplastic/malignancies. Spleen and liver status (splenomegaly and/or hepatomegaly are usually absent but can be present in neoplastic THCYT3/ET; asplenia causes thrombocythemia itself).

Laboratory testing (non-phlebotomized patients): isolated increased platelet counts $>450 \times 10^{9} / 1$ (white blood cell counts and red cell parameters are usually normal). THPO level for discrimination of primary/secondary thrombocythemia and determination of mean platelet volume (MPV) for exclusion of benign familial microcytic thrombocythemia.

Mutation analysis: THPO; MPL; JAK2 (in somatic V617F-negative cases consider germline V617I mutation).

The home page of MPN\&MPNr-EuroNet (COST Action BM0902) provides addresses of European laboratories which perform mutation analysis for hereditary thrombocythemia: http://www.mpneuronet.eu/.

\subsubsection{Describe the burden of alternative diagnostic methods to the patient}

Low: blood collection is required for mutation analyses but also for evaluation of platelet counts.

Low/medium: physical examinations (low) and additional examinations, for example, sonography/radiology (low/medium).

\subsubsection{How is the cost effectiveness of alternative diagnostic methods to be judged?}

Analysis of platelet counts, MPV and THPO levels: low costs, low/ medium sensitivity and specificity. 


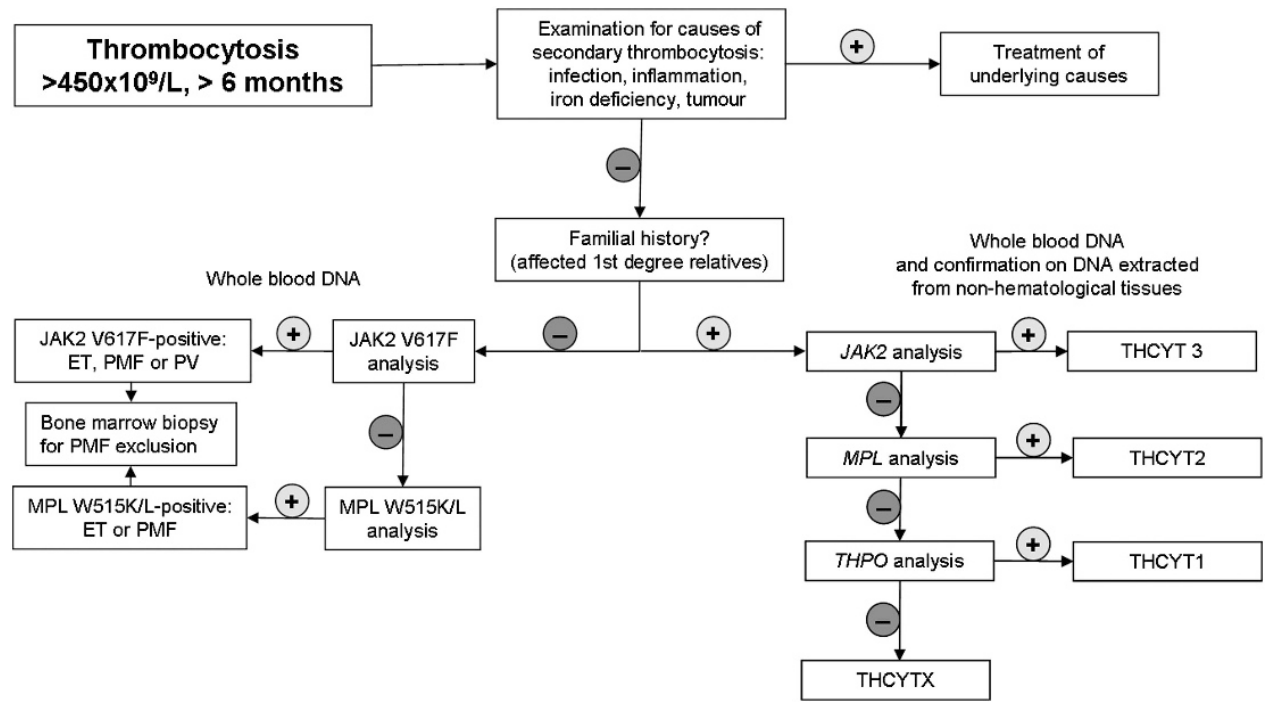

Figure 1 Proposed algorithm for evaluation of suspected THCYT.

Physical and additional examinations: depending on the examination medium to high costs, low/medium sensitivity and specificity.

3.1.4 Will disease management be influenced by the result of a genetic test?

\begin{tabular}{|c|c|}
\hline$凶$ & \\
\hline $\begin{array}{l}\text { Therapy (please } \\
\text { describe) }\end{array}$ & $\begin{array}{l}\text { Control (watch-and-wait), acetylsalicylic acid (if } \\
\text { symptomatic). }\end{array}$ \\
\hline Prognosis (please & Good prognosis, $>10$ years survival. \\
\hline & $\begin{array}{l}\text { Possible complications: thrombosis, thromboembolism } \\
\text { and hemorrhage. } \\
\text { No increased risk for progression to acute leukemia or } \\
\text { myelofibrosis in THCYT1. } \\
\text { Increased risk for progression to myelofibrosis in MPL } \\
\text { S505N-positive THCYT2. No increased risk for pro- } \\
\text { gression to acute leukemia in THCYT2. } \\
\text { Low risk for progression to myelofibrosis and/or acute } \\
\text { leukemia in THCYT3/hereditary ET; similar risk as in } \\
\text { sporadic ET } 11,20,21 \text {. }\end{array}$ \\
\hline $\begin{array}{l}\text { Management (please } \\
\text { describe) }\end{array}$ & $\begin{array}{l}\text { Provide information for the patient, drug therapy for } \\
\text { prevention of complications and control of disease } \\
\text { status. } \\
\text { Patients with the diagnosis of THCYT2 (in particular } \\
\text { MPL S505N-positive cases) and THCYT3 should be } \\
\text { monitored carefully and bone marrow biopsy as well as } \\
\text { cytogenetic analysis should be performed if progression } \\
\text { of the disease is suspected. }\end{array}$ \\
\hline
\end{tabular}

3.2 Predictive Setting: The tested person is clinically unaffected but carries an increased risk based on family history

(To be answered if in 1.10 'B' was marked).

3.2.1 Will the result of a genetic test influence lifestyle and prevention? Yes, particularly detection of JAK2 V617F or MPL S505N, because the cases might develop myelofibrosis (JAK2 V617F and MPL S505N) or acute leukemia (JAK2 V617F). ${ }^{11}$
Simultaneous factor VIII:C or von Willebrand factor antigen abnormalities with increased risk of bleeding diathesis might be present, in particular in THCYT1 and THCYT3. ${ }^{6,24}$ Compared with ET patients, THPO-mutated patients (THCYT1) have a similarly higher prothrombotic risk. ${ }^{8}$

3.2.2 Which options in view of lifestyle and prevention does a person at-risk have if no genetic test has been done?

Regular follow-up examinations.

3.3 Genetic risk assessment in family members of a diseased person (To be answered if in 1.10 ' $\mathrm{C}$ ' was marked)

3.3.1 Does the result of a genetic test resolve the genetic situation in that family?

Yes, if germline mutations can be detected (JAK2 V617I, MPL K39N, MPL P106, MPL S505N and THPO mutations).

No, if somatic (secondary) mutations are detected (JAK2 V617F and MPL mutations). Note that JAK2 and MPL mutations can be present in $\mathrm{B}$ and $\mathrm{T}$ lymphocytes without necessarily indicating germline distribution (non-myeloid differentiation of the neoplastic clone). ${ }^{25}$ Use non-hematopoietic tissue for germline analysis, for example, oral epithelia or others. ${ }^{26}$

3.3.2 Can a genetic test in the index patient save genetic or other tests in family members?

Depends on the platelet counts of other family members, but genetic testing should be performed, particularly if the index patient carries a thrombocythemia-associated mutation.

3.3.3 Does a positive genetic test result in the index patient enable a predictive test in a family member?

Yes, detection of associated mutations in a family member other than the index patient is associated with an increased risk of having/ developing thrombocythemia.

\subsection{Prenatal diagnosis}

(To be answered if in 1.10 ' $\mathrm{D}$ ' was marked)

3.4.1 Does a positive genetic test result in the index patient enable a prenatal diagnosis?

Not applicable. 


\section{IF APPLICABLE, FURTHER CONSEQUENCES OF TESTING}

Please assume that the result of a genetic test has no immediate medical consequences. Is there any evidence that a genetic test is nevertheless useful for the patient or his/her relatives?

Advantage of genetic testing:

Mutation analysis is helpful to establish diagnosis and subclassification of the disease. Mutation analysis helps to determine the risk regarding homozygous and heterozygous mutation-carrying individuals.

\section{CONFLICT OF INTEREST}

The authors declare no conflict of interest.

\section{ACKNOWLEDGEMENTS}

This work was supported by EuroGentest, an EU-FP6 supported NoE, contract number 512148 (EuroGentest Unit 3: 'Clinical genetics, community genetics and public health', Workpackage 3.2).

1 Schlemper RJ, van der Maas AP, Eikenboom JC: Familial essential thrombocythemia: clinical characteristics of 11 cases in one family. Ann Hematol 1994; 68: 153-158.

2 Wiestner A, Schlemper RJ, van der Maas AP, Skoda RC: An activating splice donor mutation in the thrombopoietin gene causes hereditary thrombocythaemia. Nat Genet 1998; 18: 49-52.

3 Liu K, Kralovics R, Rudzki Z et al: A de novo splice donor mutation in the thrombopoietin gene causes hereditary thrombocythemia in a Polish family. Haematologica 2008; 93: 706-714.

4 Kondo T, Okabe M, Sanada M et al: Familial essential thrombocythemia associated with one-base deletion in the $5^{\prime}$-untranslated region of the thrombopoietin gene. Blood 1998; 92: 1091-1096.

5 Graziano C, Carone S, Panza E: Association of hereditary thrombocythemia and distal limb defects with a thrombopoietin gene mutation. Blood 2009; 114: 1655-1657.

6 Kikuchi M, Tayama T, Hayakawa H, Takahashi I, Hoshino H, Ohsaka A: Familial thrombocytosis. Br J Haematol. 1995; 89: 900-902.

7 Ghilardi N, Wiestner A, Kikuchi M, Ohsaka A, Skoda RC: Hereditary thrombocythaemia in a Japanese family is caused by a novel point mutation in the thrombopoietin gene. Br J Haematol 1999; 107: 310-316.

8 Moliterno AR, Williams DM, Gutierrez-Alamillo LI, Salvatori R, Ingersoll RG, Spivak JL: Mpl Baltimore: a thrombopoietin receptor polymorphism associated with thrombocytosis. Proc Natl Acad Sci USA 2004; 101: 11444-11447.

9 El-Harith el-HA, Roesl C, Ballmaier M et al: Familial thrombocytosis caused by the novel germ-line mutation p.Pro106Leu in the MPL gene. Br J Haematol 2009; 144: 185-194.
10 Ding J, Komatsu H, Wakita A, Kato-Uranishi $M$ et al: Familial essential thrombocythemia associated with a dominant-positive activating mutation of the c-MPL gene, which encodes for the receptor for thrombopoietin. Blood 2004; 103. 4198-4200.

11 Teofili L, Giona F, Torti L: Hereditary thrombocytosis caused by MPLSer505Asn is associated with a high thrombotic risk, splenomegaly and progression to bone marrow fibrosis. Haematologica 2010; 95: 65-70.

12 Liu K, Martini M, Rocca B et al: Evidence for a founder effect of the MPL-S505N mutation in eight Italian pedigrees with hereditary thrombocythemia. Haematologica 2009; 94: 1368-1374.

13 Beer PA, Campbell PJ, Scott LM et al: MPL mutations in myeloproliferative disorders: analysis of the PT-1 cohort. Blood 2008; 112: 141-149.

14 James C, Ugo V, Le Couédic JP et al: A unique clonal JAK2 mutation leading to constitutive signalling causes polycythaemia vera. Nature 2005; 434 . 1144-1148.

15 Bellanné-Chantelot C, Chaumarel I, Labopin M: Genetic and clinical implications of the Val617Phe JAK2 mutation in 72 families with myeloproliferative disorders. Blood 2006; 108: 346-352.

16 Mead AJ, Rugless MJ, Jacobsen SE, Schuh A: Germline JAK2 mutation in a family with hereditary thrombocytosis. N Engl J Med 2012; 366: 96796-96799.

17 Stuhrmann M, Bashawri L, Ahmed MA et al: Familial thrombocytosis as a recessive, possibly X-linked trait in an Arab family. Br J Haematol 2001; 112: 616-620.

18 Cohen N, Almoznino-Sarafian D, Weissgarten J et al: Benign familial microcytic thrombocytosis with autosomal dominant transmission. Clin Genet 1997; 52: 47-50.

19 Bock O, Büsche G, Koop C, Schröter S, Buhr T, Kreipe H: Detection of the single hotspot mutation in the $\mathrm{JH} 2$ pseudokinase domain of Janus kinase 2 in bone marrow trephine biopsies derived from chronic myeloproliferative disorders. J Mol Diagn 2006; 8: $170-177$.

20 Percy MJ, Rumi E: Genetic origins and clinical phenotype of familial and acquired erythrocytosis and thrombocytosis. Am J Hematol 2009; 84: 46-54.

21 Teofili L, Larocca LM: Advances in understanding the pathogenesis of familial thrombocythaemia. Br J Haematol 2011; 152: 701-712.

22 Landgren O, Goldin LR, Kristinsson SY, Helgadottir EA, Samuelsson J, Björkholm M: Increased risks of polycythemia vera, essential thrombocythemia, and myelofibrosis among 24,577 first-degree relatives of 11,039 patients with myeloproliferative neoplasms in Sweden. Blood 2008; 112: 2199-2204.

23 Saint-Martin C, Leroy G, Delhommeau F et al: Analysis of the ten-eleven translocation 2 (TET2) gene in familial myeloproliferative neoplasms. Blood 2009; 114 : 1628-1632

24 Giannini S, Solimando M, Fierro T, Baronciani L, Federici AB, Gresele P: Acquired von Willebrand syndrome type 2A in a JAK2-positive essential thrombocythaemia-affected member of a large von Willebrand disease family with a novel autosomal dominant A1716P mutation. Thromb Haemost 2011; 105: 921-924.

25 Hussein K, Bock 0, Theophile $\mathrm{K}$ et al: Biclonal expansion and heterogeneous lineage involvement in a case of chronic myeloproliferative disease with concurrent MPLW515L/JAK2V617F mutation. Blood 2009; 113: 1391-1392.

26 Hussein K, Bock O, Ballmaier M et al: Familial polycythemia vera with non-germline JAK2(V617F) mutation sparing the abnormal and clonal granulopoiesis. Leukemia 2007; 21: 2566-2568. 